\title{
Immunological memory cells
}

\author{
WERONIKA RATAJCZAK ${ }^{1}$, PAULINA NIEDŹWIEDZKA-RYSTWEJ ${ }^{2}$, BEATA TOKARZ-DEPTUEA2, \\ WIESEAW DEPTUŁA ${ }^{3}$
}

${ }^{1}$ Scientific Circle of Microbiologists, Faculty of Biology, University of Szczecin, Szczecin, Poland

${ }^{2}$ Department of Immunology, Faculty of Biology, University of Szczecin, Szczecin, Poland

${ }^{3}$ Department of Microbiology, Faculty of Biology, University of Szczecin, Szczecin, Poland

\begin{abstract}
This article reviews immunological memory cells, currently represented by $T$ and $B$ lymphocytes and natural killer (NK) cells, which determine a rapid and effective response against a second encounter with the same antigen. Among T lymphocytes, functions of memory cells are provided by their subsets: central memory, effector memory, tissue-resident memory, regulatory memory and stem memory $T$ cells. Memory $T$ and $B$ lymphocytes have an essential role in the immunity against microbial pathogens but are also involved in autoimmunity and maternal-fetal tolerance. Furthermore, the evidence of immunological memory has been established for NK cells. NK cells can respond to haptens or viruses, which results in generation of antigen-specific memory cells.

$T, B$ and NK cells, which have a role in immunological memory, have been characterized phenotypically and functionally. During the secondary immune response, these cells are involved in the reaction against foreign antigens, including pathogens, and take part in autoimmune diseases, but also are crucial to immunological tolerance and vaccine therapy.
\end{abstract}

Key words: immunological memory, memory T cells, memory B cells, memory NK cells.

(Centr Eur Immunol 2018; 43 (2): 194-203)

\section{Introduction}

Immunological memory is a unique property of the immune system as it can "store" information about a stimulus and can mount an effective response when the stimulus is encountered again. This response - a secondary immune response - is quicker and stronger than the primary response. It takes a smaller stimulus to trigger a secondary response and, what is more, it occurs even after many years since the first exposure. Immunological memory is an important mechanism that protects the organism from bacteria, viruses, fungi and parasites. It plays a significant role in understanding autoimmune diseases and is one of the decisive factors of successful treatment in transplantology. It is also crucial in vaccine therapy. The secondary immune response is dependent on many subpopulations of memory cells within T and B lymphocytes and NK (natural killer) cells.

\section{T lymphocyte subpopulations, including memory $\mathbf{T}$ cells}

Currently [1], T lymphocytes are subdivided into T helper cells (Th) (Th0, Th1, Th2, Th9, Th17, Th22, Tfh - T follicular helper, nTh2 - natural Th2), cytotoxic T cells (Tc) (Tc, NKT - natural killer T cells, T $\gamma \delta$ and T CD8 $\alpha \alpha$
- IEL - intraepithelial lymphocytes, and among them natural IEL (nIEL) and induced IEL (iIEL)), regulatory T cells (Treg) (nTreg, iTreg, $\mathrm{T}_{\mathrm{R}} 1, \mathrm{iT}_{\mathrm{R}} 35, \mathrm{~T} \mathrm{CD} 8^{+}$, including $\mathrm{CD}^{+} \mathrm{CD} 122^{+}$and $\mathrm{CD} 8^{+} \mathrm{CD} 28-$, and $\mathrm{CD} 11 \mathrm{c}^{+} \mathrm{CD} 8^{+}$), exhausted/tired and anergic $\mathrm{T}$ lymphocytes, other $\mathrm{T}$ lymphocytes (naïve $\mathrm{T}$ cells, $\mathrm{CD} 4^{+}$and $\mathrm{CD} 8^{+}$) and memory T lymphocytes $\mathrm{CD}^{+}{ }^{+}$[2] and $\mathrm{CD}^{+}[3]$.

$\mathrm{CD}^{+}$memory $\mathrm{T}$ lymphocytes differentiate from naïve $\mathrm{T}$ cells towards short-lived CD4 ${ }^{+}$effector (Tem) memory $\mathrm{T}$ cells [4], whereas $\mathrm{CD} 8^{+}$memory $\mathrm{T}$ cells have a more linear development, as naïve $\mathrm{T}$ cells lose their potential to proliferate and increase that of differentiation, successively evolving to stem memory $\mathrm{T}$ cells (Tscm), central memory $\mathrm{T}$ cells $(\mathrm{Tcm})$ and finally also effector memory $\mathrm{T}$ cells (Tem) [4-6]. The activation potential of T cells is triggered with a signal transmitted by their TCR receptors and MHC class II [2] or class I molecules [3], which occur on APCs (antigen presenting cells) [2, 3]. When T lymphocytes and APCs come into contact, the former receive other signals in their immune synapses conducive to proliferation, although many types of cytokines, IL-2 in particular $[2,3]$, accompany the process. Activated T cells undergo expansion and contraction and initiate the memory pool, with $90-95 \%$ of lymphocytes undergoing apoptosis during contraction and the remaining 5-10\% differentiating into memory $\mathrm{T}$ cells $[2,3]$. Then, memory $\mathrm{T}$ cells form central

Correspondence: Prof. Beata Tokarz-Deptuła, Department of Immunology, Faculty of Biology, University of Szczecin, 3c Felczaka St.,

71-412 Szczecin, Poland, e-mail: beata.tokarz-deptula@usz.edu.pl

Submitted: 14.06.2017; Accepted: 16.02.2018 
memory $\mathrm{T}$ cells $(\mathrm{Tcm})$, effector memory $\mathrm{T}$ cells (Tem), tissue-resident memory $\mathrm{T}$ cells (Trm), regulatory $\mathrm{T}$ cells (mTreg) and stem memory T cells (Tscm) [2].

\section{Central memory $T$ cells $(\mathrm{Tcm})$ and effector memory $T$ cells $(\mathrm{Tem})$}

Central memory $\mathrm{T}$ cells occur in secondary lymphoid organs, mainly in lymph nodes and tonsils, with the following molecules on their surface: CD45RO, CCR7, CD62L, CD44, CD27, CD28, CD95, CD122 [5, 7, 8] and LFA-1 (CD11a/CD18) molecules interacting with APCs [9]. Central memory T cells (Tcm) secrete IL-2, 4, IFN $\gamma$ and TNF as well as CD40L ligand [10, 11]. Contrary to central memory $\mathrm{T}$ cells, effector memory $\mathrm{T}$ cells (Tem) inhabit chiefly non-lymphoid peripheral tissue types, including lungs, liver and intestines [8, 12], and are capable of recirculation between blood and tissue [13]. With CD45RO, CD44, CD95, CD122 molecules [7, 14] and KLRG1 protein (killer cell lectin-like receptor G1) on their surface, they do not express L-selectin (also known as CD62L) or CCR7 receptor [7]. They secrete proinflammatory cytokines, such as IL-4, 5 and IFN $\gamma$ as well as granular perforin with granzyme B [12], which confirms their cytotoxic properties $[3,12]$. The population of Tem cells with the CD4 marker, particularly with Th1 phenotype [15, 16], and CD8 receptor Tem cells [17] play an important role in chronic bacterial, viral and parasitic infections, as their effector functions are additionally strengthened by the effect of IFN $\gamma[15,18]$. In infections with Listeria monocytogenes, Salmonella typhimurium and Escherichia coli, immune functions seem to be performed by $\mathrm{CD} 8^{+}$effector memory cells, amplified by the presence of acetates, e.g. acetyl-CoA released in such infections [19]. When the respiratory system is infected with SARS or MERS viruses, its tissue is colonized mainly by $\mathrm{CD}^{+} \mathrm{T}$ cells, with a Tem cell phenotype [18]. Upon re-exposure to an antigen, even though its concentration may be very low, they activate the natural mechanisms of the immune response. These cells also facilitate migration of dendritic cells (DC) to mediastinal lymph nodes and help direct to the lungs virus specific CD8+ T lymphocytes [18]. Both CD4 ${ }^{+}$and $\mathrm{CD}^{+}$effector memory cells (Tem) were demonstrated to be involved in each secondary immune response. Such cells vary in number and $\mathrm{CD}^{+}$cells expand less than $\mathrm{CD}^{+}$cells [20]. MacLeod et al. (2010) showed that during re-infection CD4 receptor lymphocytes do not aim at directly responding to a pathogen. Instead, through secretion of cytokines their role is to support other cells involved in the immune response.

\section{Tissue-resident memory $\mathbf{T}$ cells $(\mathrm{Trm})$}

Trm cells occupy specific locations without recirculating and therefore are the first line of defense against pathogens, particularly in the digestive tract, the female reproductive system, lungs, the skin and in the brain [2123]. Their response is faster and more effective than that of other memory $\mathrm{T}$ cells migrating to the location [23]. Trm cells have surface markers such as CD69 and CD 103 [24], for which E-cadherin is a ligand, found in endothelial cells, and whose presence conditions their population in tissues [23]. The feature is also conditioned by CD49a and $\alpha_{E} \beta_{7}$ integrin, specific to intercellular bonds and adhesion structures [25]. CCR7 markers and S1P1 receptors (sphingosine-1-phosphate receptor 1), found in small quantities on the Trm cell surface, guarantee Trm cell colonization in their location. The cells are understood to have little or zero potential to recirculate [24], and similar to Tem cells they have high expression of CD44 and low levels of L-selectin [24]. Trm cells comprise $\mathrm{CD}^{+}$and $\mathrm{CD} 8^{+}$populations [21]. CD4 and CD8 Trm cells from the lungs and the brain also have CD103 receptors and show elevated levels of IFITM3 (interferon-induced transmembrane protein 3), controlled by ISG (interferon stimulated genes), which protects them from cell death by preventing these cells from infecting themselves [23].

$\mathrm{CD}^{+}$Trm cells evolve from common naive $\mathrm{T}$ cell precursors and their expansion quickly follows infection [21]. They do not express KLRG1, and only during their maturation can CD69 and CD103 markers be found on their surface [21, 25]. Their development does not depend on the presence of an antigen, but can be mediated by tissue cytokines, as is evidenced by intraepithelial Trm cells, found in the skin, in the female reproductive system and in intestines $[21,25,26]$. It is different in the case of CD8 ${ }^{+}$ Trm lymphocytes inhabiting the brain, sensory ganglia and the lungs, which do need an antigen for the maturation of CD103 Trm cells [21]. What is more, the maturation of CD8 ${ }^{+}$Trm cells involves TGF $\beta$ (transforming growth factor $\beta$ ), TNF $\alpha$ (tumor necrosis factor $\alpha$ ), IL-33 and 15 [21, 25]. $\mathrm{CD}^{+}$Trm lymphocytes isolated from the brain, skin and intestinal mucosa exhibit increased effector function as they are conducive to more intensive and faster synthesis of IFN $\gamma$, which facilitates the lysis of target cells [27]. The effect of $\mathrm{CD}^{+}$Trm on viruses seems to be stronger than that of common Tc lymphocytes [27]. Activated CD8 ${ }^{+}$ Trm cells initiate antimicrobial defense, for example by inducing expression of antibacterial and antiviral genes, and facilitate the activity of APCs $[21,27]$. CD8 ${ }^{+}$Trm cells were shown to activate NK cells and maturation of dendritic cells and affect migration of $\mathrm{B}$ and $\mathrm{T}$ cells to the location of infection using pathways dependent on IFN $\gamma$, TNF and IL-2R [21, 27]. The increase of the CD8 ${ }^{+}$Trm population in the CNS (central nervous system) of an aging organism was demonstrated [28] to weaken TNF production and to strengthen phagocytic activity of microglia in the CNS, which can slow down the aging processes of the brain.

Although the role of $\mathrm{CD}^{+}$Trm lymphocytes in tissue immune function has been less understood than that of $\mathrm{CD}^{+}$Trm lymphocytes, they seem to be equally im- 
portant in the immune response in locations they occupy. CD4 ${ }^{+}$Trm lymphocytes commonly occur in non-lymphoid tissues, especially in the dermis. Their presence has been confirmed in the lungs, genital mucosa, intestines [24, 29, 30] and even in the salivary glands [29]. CD4 ${ }^{+}$Trm and $\mathrm{CD}^{+}$Trm cells are involved in the direct immune response and mediate how other cells migrate to tissues [30]. CD69 and CD11a markers were found on the surface of $\mathrm{CD}^{+}$ Trm cells in mice and the Trm cell defining CD103 marker occurs only in cells populating the skin [30]. CD4 ${ }^{+}$Trm lymphocytes demonstrate the ability of a rapid immune response, and, like $\mathrm{CD}^{+}{ }^{+}$Trm secrete IFN $\gamma$ and IL-17 in the mucosa [30]. Research conducted on a mouse model [31] showed that, through IFN $\gamma$ secretion and $\mathrm{CD}^{+} \mathrm{T}$ cell recruitment (Te, Tem and Tcm) to the site of infection, $\mathrm{CD}^{+}$ Trm cells induce immunity in Leishmania major infections and are present in the skin, regardless of whether or not an antigen is present [31]. The cells were demonstrated [32, 33] to provide effective protection in successive infection of lungs with the influenza virus. For a short time after infection onset, they are able to produce different cytokines, including IFN $\gamma$ [33].

In people infected with herpes viruses (HSV-1 and HSV-2), which e.g. infect vaginal mucosa in women, CD4 ${ }^{+}$ Trm lymphocytes and CD8 ${ }^{+}$Trm cells [27] occur in large numbers, conditioning their fighting potential against infected cells through massive granzyme B secretion [23]. These cells are required to maintain skin homeostasis, as they release IL-15 and IL-17 in hair follicles [34]. IL-15 was shown to be a key element in keeping CD8 ${ }^{+}$ Trm cells in the epidermis, with IL-17 playing a similar role for $\mathrm{CD}^{+}$and $\mathrm{CD}^{+}$Trm cells [34]. The role of $\mathrm{CD}^{+}$ and $\mathrm{CD} 4^{+}$Trm cells in the course of psoriasis, baldness, albinism and transplant rejection was demonstrated [35]. The cells can be one of the causes of cutaneous T-cell lymphoma and its variants: mycosis fungoides and Sézary syndrome [35], although it is suggested that they play an important role in pathology of hard tumors [36]. Additionally, apart from $\mathrm{CD}^{+}$and $\mathrm{CD} 8^{+}$Trm cells, the potential to fight off infection is also provided by Treg lymphocytes, $\gamma \delta \mathrm{T}$ cells, natural killer T cells (NKT), including intraepithelial ones [24, 29] and innate lymphoid cells (ILC) [37]. Recent findings have shown that group 2 innate lymphoid cells (ILC2s) can be classified as immunological memory cells. Moreover, these cells share many features with memory T lymphocytes and memory NK cells [38].

\section{Regulatory T lymphocytes (Treg), including memory regulatory $\mathbf{T}$ cells (mTreg)}

Regulatory $\mathrm{T}$ lymphocytes comprise a subpopulation of diverse lymphocytes involved in maintaining immune homeostasis of an organism, in modulating the inflammatory response to antigens, in preventing autoimmune reactions and in inhibiting chronic infections [39-41]. Treg cells are also involved in the immune response in cancer development [42] and in immunosuppressive processes in grafting organs and tissues [43, 44]. Treg cells can be subdivided into natural regulatory $\mathrm{T}$ (nTreg) cells that are formed in the thymus and induced Treg (iTreg) cells which form in secondary lymphoid organs [45]. Moreover, nTreg cells can be subdivided into pTreg cells which develop peripherally and tTreg cells which develop in the thymus [46, 47]. tTreg cells make up a substantial part of mTreg cells and when activated with antigens in non-lymphoid tissues, they express Helios protein and neuropilin-1 (NRP1). Upon the second exposure to an antigen, they tend to inhibit autoimmune reactions [47]. pTreg lymphocytes in intestines prevent an immune reaction against local commensal bacteria [47]. Apart from nTreg cells, which are subdivided into tTreg, pTreg and iTreg, the Treg group also comprises $\mathrm{T}_{\mathrm{R}} 1$ and $\mathrm{iT}_{\mathrm{R}} 35$ lymphocytes [1]. The former $-T_{R} 1-$ are cells without the FOXP3 marker, although CD3 and CD4 markers are found on their surface. They express ROG, a repressor of GATA-3, responsible for inhibition of their effector function and production of cytokines [1]. $T_{R} 1$, like $\mathrm{iT}_{R} 35$ lymphocytes, do not express the FOXP3 marker but they produce substantial amounts of IL-10. $\mathrm{iT}_{\mathrm{R}} 35$ cells release IL-35 although they do not produce TGF $\beta$, common to Treg lymphocytes [1]. $T_{R} 1$ cells are involved in immunotolerance, inflammatory and allergic processes as well as in bacterial and viral infections [1]. $\mathrm{iT}_{\mathrm{R}} 35$ cells display strong suppressive properties in infections and cancer [1]. All Treg lymphocytes can be subdivided into cells with CD4 and CD8 markers [48, 49]. $\mathrm{CD}^{+}{ }^{+}$Treg cells also have the CD25 receptor $[48,50]$ and CTLA4 [40] and GITR [50] molecules, although their key marker is FOXP3 [51, 52]. Treg cells make up a subpopulation of nTreg cells [46], but more subpopulations are also defined [51] as they contain other markers, such as COS, TNFR2, OX40, 4-1BB, LAG-3, LAP, GARP and to a smaller degree CD127, CD6, CD26 and CD49d [41, 53].

Similarly, $\mathrm{CD}^{+}$Treg cells have their subpopulations owing to their respective markers: $\mathrm{CD} 8^{+} \mathrm{CD} 122^{+}$, $\mathrm{CD}^{+} \mathrm{CD} 28^{-}, \mathrm{CD}^{+}{ }^{+} \mathrm{CD} 103^{+}[44]$ and $\mathrm{CD} 11 \mathrm{c}^{+} \mathrm{CD} 8^{+}[49$, 54]. Those with $\mathrm{CD} 8^{+} \mathrm{CD} 25^{+} \mathrm{FOXP} 3^{+}$receptors form a very small subpopulation without a name [55]. These specific molecules occur on CD8+ Treg cells, define their phenotype, divide them into subpopulations and functionally bring them closer to Tcm cells [44, 56, 57]. For example, cells with $\mathrm{CD} 8{ }^{+} \mathrm{CD} 122^{+}$phenotype, which is compatible with Tcm cell phenotype, owing to their functions (transplant rejection inhibition) are classified as Treg cells [44, 56]. The regulatory properties of $\mathrm{CD}^{+} \mathrm{CD} 122^{+}$Treg cells include secretion of immunosuppressive cytokines (IL-4, IL-10), transforming growth factor- $\beta$ (TGF $\beta$ ) [44] and regulating homeostasis through inducing apoptosis of activated $\mathrm{T}$ cells via interaction of Fas-FasL [56]. Importantly, the population of $\mathrm{CD} 8{ }^{+} \mathrm{CD} 122^{+}$Treg cells is characterized by different expression of the markers, which enables it to 
fulfill both Treg and Tcm cell functions [58]. Despite the fact that $\mathrm{CD} 8{ }^{+} \mathrm{CD} 122^{+}$Treg and $\mathrm{CD} 8^{+} \mathrm{CD} 122^{+} \mathrm{Tcm}$ cells have the same receptors, they differ from one another in the presence or the lack of the PD-1 (programmed death receptor-1) molecule which is responsible for suppression of autoimmunity and for facilitating tolerance to antigens [57, 59].

While discussing Treg lymphocytes, including CD4 ${ }^{+}$ and $\mathrm{CD}^{+} \mathrm{mTreg}$ cells occurring in the skin, it was demonstrated that they express molecules typical for effector memory cells (Tem), i.e. the following markers: CD127, CD44, CD27, CCR6, and to a lesser degree the presence of L-selectin (CD62L) [61] and CD45RO receptor [62, 63]. Additionally, most $\mathrm{CD}^{+}$and $\mathrm{CD}^{+}$mTreg cells found in the lamina propria of mucosa in the small intestine had small concentrations of CD44 and CD45RB markers, which in terms of phenotype brings them closer to Tem cells [64]. It was also demonstrated that mTreg lymphocytes with a phenotype compatible with that of Tem cells can quickly migrate to non-lymphoid tissues, such as the liver and the lungs. Owing to that, they prevent these tissues from being damaged in a secondary infection [65]. Additionally, in successive viral infection, mTreg lymphocytes release substantial amounts of anti-inflammatory cytokines, e.g. IL-10, thereby controlling the excessive influence of $\mathrm{CD}^{+} \mathrm{T}$ lymphocytes $[39,64]$. They were confirmed to be involved in a reaction against commensal bacteria occurring in human skin [66]. Although questionable, the role of mTreg cells was confirmed in pregnancy, during which the mother's body requires tolerance of the genetically different parental set of antigens in the fetus [67]. This goes back to the fact that mTreg cells formed in the first pregnancy display antigen specificity to fetal and parental antigens, and when pregnancy is terminated, they remain in the mother's body at a low level [68]. However, upon re-exposure to fetal antigens during the second pregnancy, they can quickly reproduce a population of Treg cells [66-69]. Through the immune tolerance of fetal tissues, the cells eliminate their rejection and therefore prevent miscarriage, premature birth and pre-eclampsia that may occur in successive pregnancies $[69,70]$. Despite all these facts, the cells are not classified as "real" immune memory cells during pregnancy. Instead, they are understood to be a population of cells maintained at a constant, if low, level, which is the result of antigen stimulation of fetal cells which in the post-pregnant mother create microchimerism [67].

\section{Memory $\mathbf{T}$ cells with stem cell-like properties (Tscm)}

Tscm cells, like stem cells, have a great potential to regenerate themselves [6]. In the general population of $\mathrm{CD}^{+}$and $\mathrm{CD}^{+} \mathrm{T}$ cells, Tscm cells are found in small quantities and their phenotype is similar to that of naïve
T cells. The following molecules are to be found on their surface: CD45RA, CCR7, CD27, CD62L (L-selectin), CD95, CD122 (markers of other memory cells), and SCA1, BCL-2 (B-cell lymphoma 2), IL-2R $\beta$ receptor [6], CCR3 and CCR4 [5, 7]. There is a lack of or small quantities of the following markers: CD45RO [5, 67], CD38, CD31 [5] CD44 [6] and CD95 present on their surface through interaction with the Fas ligand induces cell apoptosis. However, when CD95 interacts with transcription factor TCF (T-cell factor), the $\beta$-catenin signal pathway is activated, which plays a role in the cell self-renewal process [6]. Il-7 and 15 are factors facilitating expansion and unlimited generation of Tscm cells [5]. Tscm lymphocytes, like Tcm and Tem cells, have the ability of a quick response following stimulation with viral antigens and can respond to autoantigens generated in tumorigenesis [6].

\section{Subpopulations of B lymphocytes, including memory $B$ cells}

B cells can be subdivided into B1 and B2 lymphocytes $[11,71]$, IRA B cells (innate response activator B cells) $[71,72]$, regulatory $B$ (Breg) lymphocytes and memory $\mathrm{B}$ cells $(\mathrm{Bm})[71,73]$.

B1 (B1a and B1b) lymphocytes differentiate from precursor cells in fetal liver and do not occur in the bone marrow of adult humans [11]. CD19, B220, IgM, CD43 molecules and expression of the CD5 marker are found on their surface, which regulates the B1a subpopulation, as those without the CD5 marker form a B1b subpopulation [71]. B2 lymphocytes occur in adults, evolve from bone marrow stem cells and recirculate between blood and secondary lymphoid organs [11]. Apart from expressing markers specific for B1 cells, i.e. CD19, B220, IgM, CD43, they have also CD21 and CD23 markers, forming a subpopulation of follicular B2 cells [71], as those without CD21 and CD23 are classed as marginal zone (MZ) B2 lymphocytes and occur in the marginal zone of the white pulp of the spleen [71].

The third population of B lymphocytes comprises innate response activators (IRA), a subset related to B1a cells [71]. The cells express on their surface CD43, CD23, CD93 markers and display high expression of $\operatorname{IgM}$. They can also produce GM-CSF (granulocyte-macrophage colony-stimulating factor) [71], which activates monocytes, macrophages and neutrophils [72]. The fourth population of B lymphocytes comprises Breg cells, which are characterized by substantial phenotypic diversity, as they are made up of many cell subpopulations [71, 73]. In humans and mice, subpopulations of cells have been isolated among regulative B lymphocytes [74]. Breg cells in mice were subdivided into seven subtypes based on the suppression mechanism, meaning IL-10 production, and these are T2-MZP B lymphocytes (transitional 2-marginal zone precursor cells), marginal zone B lymphocytes, B10 and 
B-1a cells, GIFT-15 cells, plasmablast and TIM-1 ${ }^{+}$B cells. In addition, the subpopulation of Breg is distinguished, in which IL-35 produced by them contributes to the suppression of the immune response. It has also been found that the mouse Breg family includes killer B lymphocytes, expressing the Fas ligand (FasL) and one expressing PD-L1 programmed death receptor-1. Breg cells are also eligible for the $\mathrm{B} 220^{+} \mathrm{CD} 39^{+} \mathrm{CD} 73^{+}$phenotype, in which adenosine is also involved in the suppression mechanism, which also participates in T-cell effector suppression [74]. In contrast, in humans [74], seven subtypes of Breg lymphocytes have also been distinguished, which show a much more diverse mechanism of suppression of the immune response than mice. Human regulatory B cells that secrete IL-10 are B10 cells and plasmablasts with $\mathrm{CD} 27^{\text {int }} \mathrm{CD} 38^{\text {hi }}$ phenotype and immature $\mathrm{B}$ cells with $\mathrm{CD} 19^{+} \mathrm{CD} 24^{\mathrm{hi}} \mathrm{CD} 38^{\text {hi }}$ phenotype that act by secretion of IL-10 and based on expression of PD-L1. The fourth Breg subpopulation in humans consists of Br1 lymphocytes acting by secretion of IL-10 and allergen-specific IgG4 antibodies and which furthermore participate in inhibiting $\mathrm{CD}^{+}{ }^{+} \mathrm{T}$ cell proliferation. The other subtypes of human Breg lymphocytes were distinguished based on the ability to express granzyme B, indoleamine 2,3-dehydrogenase (IDO) $\left(\mathrm{GrB}^{+} \mathrm{B}\right.$ cells) and TGF- $\beta$ (iBregs). Among human Breg, as in mice, mucosal subtypes have been identified that express ectoenzymes and receptors CD39 and CD73 responsible for adenosine dephosphorylation catalysis [74]. They display immunosuppressive and modulating functions and through release of IL-10 and 35, TGF $\beta$ factor, together with T cells, NKT, monocytes and DC, they affect the immune response [73, 75]. These secreted cytokines affect the reduction of APC cell function and decrease the effector $\mathrm{T}$ cell response and Treg induction [75]. In addition, Breg lymphocytes can induce apoptosis of target cells by intercellular mechanisms as a result of the ligand of FasL and PD-L1 [75]. It is believed that the main function of Breg lymphocytes associated with IL-10 production is associated with inhibition of inflammation and homeostasis, and the role of IL-35 in autoimmune disease, including autoimmune encephalomyelitis (EAE) [75]. In turn, the role of Breg cells through the TGF $\beta$ factor produced by these lymphocytes is associated with the development of Treg lymphocytes, which is reported to be a factor for diabetes, allergic diseases, colitis, and the development of tolerance to transplantation [76]. It has also been shown that Breg lymphocytes, by secretion of large amounts of IL-10, may be essential for the course of pregnancy and prevention of inflammation as a result of inhibition of monocytes and macrophages, as well as the suppression of secretion of proinflammatory cytokines and chemokines [76]. Studies have shown that in the context of Breg lymphocytes, the presence of these cells within the tumor as well as in the lymph nodes leads to immunosuppression, causing inhibition of the immune response and subsequent tumor cell proliferation and metastasis [76].

\section{Memory B lymphocytes}

Bm lymphocytes are cells involved in the secondary innate humoral immune response. They also, like other B cells, produce antibodies after the first exposure with an antigen and then produce large amounts of antibodies shortly after another exposure to the same antigen [77]. The secondary innate humoral immune response, upon the second exposure to the antigen, relies on quick production of antibodies with changed isotypes, with high affinity of $\mathrm{BCR}$ (B cells receptor) to the antigen [78]. Memory B cells can be formed in two T cell-dependent mechanisms: in the first, they differentiate into short-lived plasma cells [77, 79] and in the second, they are formed and differentiate in dependent or independent germinal centers of peripheral lymphoid organs [78]. In these secondary lymphoid organs, once B and T cells have migrated, together with $\mathrm{DC}$, the presentation of an antigen takes place. Activated cells migrate to lymphoid nodules where at their boundary zone their interaction occurs to verify affinity of antigens presented by DC [78]. In the next phase, activated B lymphocytes undergo proliferation and some of them differentiate into short-lived antibody-releasing plasma cells and some develop into memory B cells, independent of GC sites and finally others migrate to differentiation sites [78, 80]. In the case of cells that have not been included in GC sites, B cell differentiation $[78,80]$ can start from activated CD28 and GL7-expressing precursor cells [78, 80]. In differentiation sites, in the dark zone, clonal expansion of antigen-specific B cells and BCR differentiation take place $[78,80]$. Some B cells from the dark zone of GC migrate to the light zone, where, together with FDC (follicular dendritic cells) and Tfh lymphocytes, there occurs differentiation of memory B cells into GC site dependent memory cells and long-lived plasma cells. Some cells migrate back to the dark zone of differentiation sites $[78,80]$. Differentiation of memory B lymphocytes in GCs can be regulated by signals produced by Tfh cells, i.e. the following proteins: CD40L [78], SAP (SLAM-associated protein), ICOS (inducible T cell costimulator) and BCL-6 (B cell lymphoma 6) [80]. Bm cells are characterized by changing metabolism, from anabolic to catabolic, which reduces requirements for growth factors, i.e. cytokines [78]. Bm lymphocytes express BCL-2, an apoptosis preventing protein. Their production of BIM/BCL2L11 (BCL-2-like protein 11) decreases [78]. Bm lymphocytes can be produced not only during the $\mathrm{T}$ cell regulated immune response, but also from conventional marginal B2 lymphocytes, in the marginal zone of spleen white pulp [78]. B1 lymphocytes are also involved in Bm cell generation as B1 lymphocytes are most numerous in the peritoneal cavity and to a lesser degree present in the spleen, where they are responsible for identification of both their own cellular components and bacterial antigens [78]. B1 cells, together with B2 marginal zone cells, regulate the primary synthesis of IgM natural 
antibodies [81-84]. It was also demonstrated that B1a and B1b lymphocytes can produce memory cells, since B1a cells form immune memory towards glycolipid (FtL) from Francisella tularensis and B1b cells form memory pools of Streptococcus pneumoniae and Borrelia hermsii [78]. $\mathrm{Bm}$ cells evolved from differentiating B1a and B1b cells were found mainly in the peritoneal cavity, but also in the spleen, tonsils and peripheral blood [79]. Bm cell formation is dependent on hereditary and epigenetic regulations [85] - following gene expression controlled by DNA methylation, histone modifications or interaction of non-coding miRNA, plasma cells and Bm lymphocytes can be produced from B lymphocytes [85]. The Pax5 and Spib genes are expressed in memory B cells, which may be related to their differentiation from naïve $\mathrm{B}$ cells. The origin of $\mathrm{Bm}$ cells is also regulated by the presence of some enzymes, such as methyltransferase, acetyltransferase [85] and IL-10, which facilitates differentiation of plasma cells and antibody secretion from memory B cells [86].

Such diverse mechanisms of Bm cell formation mean that they comprise many types of lymphocytes, which differ from one another in their properties. The classic Bm cell population comprises $\operatorname{IgM}^{+}$B cells, with CD19, CD27 and CD80 markers on surface. They originate in GCs $[79,86]$. These lymphocytes are able to change antibody class and to differentiate into long-lived plasma cells [10]. They play an important role in immunity against bacteria producing polysaccharide capsules [79] and in forming immune memory of MSP1 (merozoite surface protein 1) Plasmodium chabaudi [87]. Research confirmed that in successive infection with $P$. chabaudi, $\operatorname{IgM}^{+} \mathrm{Bm}$ cells belonging to mice that were formed after the first exposure to the antigen were able to differentiate into antibody-synthesizing cells, i.e. T cell-regulated plasmablasts, and into plasma cells, independent of $\mathrm{T}$ lymphocytes [87]. Other memory B cells are $\mathrm{IgG}^{+} \mathrm{CD} 27^{+} \mathrm{Bm}$ cells, among which there are $\mathrm{IgG} 1, \mathrm{IgG} 2, \mathrm{IgG} 3$ and $\mathrm{IgG}^{+} \mathrm{CD} 27^{-}$memory cells, with subclasses of IgG1 and IgG3 [79], and also $\operatorname{IgA}^{+}$[79] and $\mathrm{IgE}^{+} \mathrm{Bm}$ cells [78]. $\mathrm{IgM}^{+} \mathrm{CD} 27^{-}$Bm cells were found in human tonsils. They express FCRL4 (Fc receptor-like-4) and are able to quickly differentiate into plasma cells. They are closely linked to MALT (mucosal associated lymphatic tissue) [79]. Their presence and significance was demonstrated in chronic hepatitis $\mathrm{C}$ and malaria [78]. Another study describes the role of Bm cells present in Peyer's patches in adaptation of secretory immunoglobulin A (SIgA) antibodies to intestinal microflora. Therefore, it is assumed [88] that Bm cell populations guarantee the stability of the immune system response of the host against its own microflora. The study [88] also showed that plasma cells present in mammary glands can be produced from Bm cells, which originated following the primary immune response. It explains why antibodies present in mother's milk can recognize intestinal bacterial antigens [88]. Bm lymphocytes with CD38, CD73, CD80, CD273, CD69 and CXCR3 molecules on the surface display affinity to lung tissue. During influenza virus reinfection, their populations inhabiting the lungs can quickly differentiate into plasma cells secreting IgG and IgA, antibodies neutralizing influenza hemagglutinin. That provides a quick immune response to the pathogen [89]. Bm cells were shown to play a role in the response to stimulation with monovalent hepatitis $B$ vaccine [10].

Research into Bm lymphocytes showed two subclasses within so-called TLM B (tissue-like memory B cells) [90], which express FCRL5 (Fc receptor-like-5). It is a cell population in a state of exhaustion and directed to apoptosis [90]. These cells [90] are able to produce protective antibodies in malaria, and their involvement in chronic inflammatory diseases is suggested [90]. To sum up, the main features of Bm cells are their potential for a very long "life" and an extensive response in the secondary exposure to an antigen, which is crucial in successful application of vaccines [78]. The ability of Bm cells to mount a quick secondary response results from their potential to differentiate into plasma cells, and particularly into $\mathrm{IgG}^{+} \mathrm{Bm}$ cells, which are effective in fighting off infection [86]. Another important property of $\mathrm{Bm}$ cells is that they are long-lived, which is regulated by follicular dendritic cells (FDCs) [91]. In the case of IgG2 $\mathrm{a}^{+}$and $\operatorname{IgA} \mathrm{A}^{+} \mathrm{Bm}$ lymphocytes, their survival also depends on T-bet (T-box transcription factor) $[78,92]$, crucial for Th1 development and functioning, and $\mathrm{ROR} \alpha$ (retinoic acid receptor-related orphan receptor $\alpha)[78,92]$. T-bet, expressed in B cells through pathways regulated by STAT1 (signal transducer and activator of transcription 1) and ETS1 (transcription factor), is the key factor regulating survival of $\mathrm{IgG} 2 \mathrm{a}^{+} \mathrm{Bm}$ cells and their migration to sites of inflammation [92].

\section{Subpopulations of NK cells, including memory NK cells}

NK cells are basically cells of the innate immune system and are crucial in the defense of the host against pathogen-induced infections, mainly viral infections. They are also involved in fighting off cancerous cells. Due to their ability to release inflammatory cytokines, growth factors, and chemokines NK cells can kill infected and neoplastically transformed cells [93-95]. NK cells originate in bone marrow stem cells. They are characterized by CD16 and CD56 markers [1] and their expression differentiates them into two main subpopulations: NK1 and NK2 [96]. NK1 cells show moderate expression of CD56 and a high level of CD16. Their phenotype is defined as CD56 ${ }^{\mathrm{dim}} \mathrm{CD} 16^{+}$and they are considered to be mature NK cells, which inhabit mainly blood and the spleen [95, 97]. The CD122/132 (IL-2R $\beta$ ) marker and CXCR1, CX3CR1 and ChemR23 chemokine receptors are found on their surface [98]. The cells secrete perforin, a cytolytic factor [97], and have much greater cytotoxic potential than NK2 cells [97, 98]. 
The latter display high expression of the CD56 marker, with no CD16 molecules on their surface. Their phenotype is defined as CD56 ${ }^{\text {high }} \mathrm{CD} 16$. They much more commonly inhabit secondary lymphoid organs, in particular lymph nodes and tonsils [95, 97]. CD117/c-kit, CD25 (IL-2R $\alpha$ ), IL18R $\alpha$ [98], CCR7 chemokine receptor and L-selectin (CD62L), found on the surface, regulate their migration from blood to secondary lymphoid organs $[97,98]$. On both types, i.e. NK1 and NK2, there are natural cytotoxicity receptors (NCRs), including NKp46, Nkp30, NKp44 [97, 99], NKG2D (natural killer group 2, member D) receptors and DNAM1 (DNAX accessory molecule 1), an adhesion molecule which can identify molecules formed under stress (danger) and upon exposure to viral antigens [15] - VSV (vesicular stomatitis virus), VLPs (virus-like particles) - the influenza virus hemagglutinin and Gag and Env proteins of the HIV-1 virus [100]. The cells also express receptors not regulated by RAGs (recombination-activating genes), which are responsible for their interaction with pathogens or stress-induced auto-antigens [101]. NK cells, on their surface also have killer Ig-like receptors (KIR) and CD94/NKG2A molecules, which identify HLA class I histocompatibility antigens $[97,99]$. NK cells may be induced by cytokines, e.g. IFN $\alpha$ and IL-12, 15 and 18 [96]. Their cytotoxic properties are linked to granularity and secretion of large quantities of IFN $\gamma$ and other growth factors, such as TNF, GM-CSF and the CCL4 ligand, which significantly regulates the immune response [99].

\section{Memory NK cells}

Currently, NK cells are thought to have a cell subset with memory properties, following stimulation with haptens or viruses [102-104] and generated independent of antigens, only as a result of "memory" of proinflammatory cytokines [102, 104-106]. Research conducted on mice [107] showed that 70 days after infection with murine cytomegalovirus (MCMV) NK cells were identified in the mice. The cells have on the surface, similar to naïve $\mathrm{NK}$ cells, the $\mathrm{Ly} 49 \mathrm{H}^{+}$receptor, the difference being that in memory NK cells the expression of the receptor is much higher [107]. The lymphocytes show high expression of KLRG1, CD43, Ly6C molecules and slightly lower expression of CD27, which phenotypically brings them closer to common memory T cells [107]. "Memory-like" NK cells are specific to 157 viral factor, a protein of MCMV (mouse cytomegalovirus) [104, 106, 108-110]. They are also sensitive to proinflammatory cytokines secreted by DC [106]. The cells exhibit much higher expression of IFN $\gamma$ coding genes [107], which indirectly regulates their cytotoxicity, e.g. in infections with human influenza A virus [111]. They populate not only the liver, but also blood, the spleen and kidneys [111] and may recreate a pool of antigen specific cells [107]. Memory NK cells were found to display specificity towards CXRC6-regulated haptens in the liver [108, 109, 112]. Although CXRC6 is not required for the cells to be able to identify antigens, it seems to be involved in maintaining the effector functions of memory NK cells and ensures their survival [112]. The immune response, with the involvement of NK cells, was demonstrated on the skin and in tissues in contact with viral antigens [113]. NK cells found in pleural effusion in patients infected with Mycobacterium tuberculosis contained the CD45RO marker, which shows that the cells have phenotypic affinity to memory T lymphocytes. The presence of the CD45RO marker seems to play a role in amplifying the immune response, in which IL-12-induced NK cells (phenotypically similar to memory T cells) release larger amounts of IFN $\gamma$ [114]. IL-12 activates STAT4 (signal transducer and activator of transcription 4), involved in expansion and formation of the cells [109]. Apart from memory NK cells being present following the response to specific viral antigens, cytokine-dependent memory NK cells were found, independent of the presence of antigens [93]. Research conducted on mice showed [115] that NK cell activation with IL-12 and 18 in the presence of IL-15 resulted in formation of an NK lymphocyte population able to release IFN $\gamma$ [102, 106, 115]. Additional activation with IL-12, 18 and 15 led to formation of memory NK cells and their daughter cells. Research $[115,116]$ suggests that the mechanism of cell generation does not require their cell cycle to be performed, which makes their "memory" seem to be hereditary. Formation of NK cells also involves BCL-2 (B-cell lymphoma 2) and BIM/BCL2L11 (BCL-2-like protein 11) - proteins active in apoptosis and BNIP3 (Bcl-2 nineteen-kilodalton interacting protein 3) and BNIP3L (BNIP3-like) - proteins active in autophagy [93, 109]. It was demonstrated that even in undisturbed homeostasis, exposure of NK cells to cytokines resulted in formation of memory NK cells [115]. Memory NK cells in humans and in mice show a different phenotype conditioned by factors that activate them $[105,116]$. Human memory NK cells express CD94/NKG2C [102, 111, 116], CD57 [105, 111], CG69, SYK, EAT2 and KIRD2DL markers [105]. Memory NK cells in mice express THY1, LY49C or LY491 and CXCR6 [103], LY49H, CD11b, KLRG1 and LY6C. The cells exhibit low expression of CD27 [107], although some research [105] found NKp46, DX5 and CD43 markers.

\section{Conclusions}

Research on memory cells of the immune system has been crucial to the discovery of new subpopulations of immune system cells and to the understanding of their new functions. It also sheds light on their as yet unknown role in physiological and pathological states. New data on the scope of immunological memory may be instrumental in developing new standards of treatment for many diseases, including autoaggressive diseases and cancer.

The authors declare no conflict of interest. 


\section{References}

1. Niedźwiedzka-Rystwej P, Tokarz-Deptuła B, Deptuła W (2013): Charakterystyka subpopulacji limfocytów T. Postepy Hig Med Dosw 67: 371-379.

2. Gasper DJ, Tejera MM, Suresh M (2014): CD4 T-cell generation and maintenance. Crit Rev Immunol 34: 121-146.

3. Valbon SF, Condotta SA, Richer MJ (2016): Regulation of effector and memory CD8+ T cell function by inflammatory cytokines. Cytokine 82: 16-23.

4. Golubovskaya V, Wu L (2016): Different subsets of T cells, memory, effector functions and CAR-T immunotherapy. Cancers 8: 36 .

5. Flynn JK, Gorry PR (2014): Stem memory T cells (TSCM) - their role in cancer and HIV immunotherapies. Clin Transl Immunology 3: 1-7.

6. Gattinoni L, Lugli E, Ji Y, et al. (2011): A human memory T cell subset with stem cell-like properties. Nat Med 17: 12901297.

7. Busch DH, Fräßle SP, Sommermeyer D, et al. (2016): Role of memory $\mathrm{T}$ cell subsets for adoptive immunotherapy. Semin Immunol 28: 28-34.

8. Sallusto F, Geginat J, Lanzavecchia A (2004): Central memory and effector memory $\mathrm{T}$ cell subsets: function, generation and maintenance. Annu Rev Immunol 22: 745-763.

9. Faint JM, Annels NE, Curnow SJ, et al. (2001): Memory T cells constitute a subset of the human CD8+CD45RA+ pool with distinct phenotypic and migratory characteristics. J Immunol 167: 212-220.

10. Rosado MM, Scarsella M, Pandolfi E, et al. (2011): Switched memory B cells maintain specific memory independent of serum antibodies: The hepatitis B example. Eur J Immunol 41: 1800-1808.

11. Sage AP, Mallat Z (2014): Multiple potential roles for B cells in atherosclerosis. Ann Med 46: 297-303.

12. Ahlers JD, Belyakov IM (2010): Memories that last forever: strategies for optimizing vaccine T-cell memory. Blood 115: 1678-1689.

13. Zhang Q, Lakkis FG (2015): Memory T cell migration. Front Immunol 6: 504.

14. Roberts AD, Ely KH, Woodland DL (2005): Differential contributions of central and effector memory $\mathrm{T}$ cells to recall responses. J Exp Med 202: 123-133.

15. Borges de Silva H, Fonseca R, Alvarez JM, et al. (2015): IFN $\gamma$ priming effects on the maintenance of effector memory CD4+ T cells and phagocyte function: evidence from infectious disease. J Immunol Res 3: 1-8.

16. Tubo NJ, Fife BT, Pagan AJ, et al. (2016): Most microbe specific nadve CD4+ T cells produce memory cells during infection. Science 351: 511-514.

17. Martin MD, Kim MT, Shan Q, et al. (2015): Phenotypic and functional alterations in circulating memory CD8 T cells with time after primary infection. PLoS Pathog 11: e1005219.

18. Zhao J, Zhao J, Managalam AK, et al. (2016): Airway memory $\mathrm{CD}^{+} \mathrm{T}$ cells mediate protective immunity against emerging respiratory coronaviruses. Immunity 44: 1379-1391.

19. Balmer ML, Ma EH, Bantug GR, et al. (2016): Memory CD8+ $T$ cells require increased concentrations of acetate induced by stress for optimal function. Immunity 44: 1-13.

20. MacLeod MKL, Kappler JW, Marrack P (2010): Memory CD4 T cells: generation, reactivation and re-assignment. Immunology 130: 10-15.
21. Carbone FR (2015): Tissue-resident memory T cells and fixed immune surveillance in nonlymphoid organs. J Immunol 195: 17-22.

22. Clark RA (2015): Resident memory T cells in human health and disease. Sci Transl Med 7: 1-8.

23. Shin H, Iwasaki A (2013): Tissue-resident memory T cells. Immunol Rev 255: 165-181.

24. Mueller SN, Mackay LK (2016): Tissue-resident memory T cells: local specialists in immune defence. Nat Rev Immunol 16: 79-89.

25. Mackay LK, Rahimpour A, Ma JZ, et al. (2013): The developmental pathway for CD103+CD8+ tissue-resident memory T cells of skin. Nat Immunol 14: 1294-1301.

26. Casey KA, Fraser KA, Schenkel JM, et al. (2012): Antigen independent differentiation and maintenance of effector-like resident memory $\mathrm{T}$ cells in tissues. J Immunol 188: 48664875 .

27. Thom JT, Oxenius A (2016): Tissue-resident memory T cells in cytomegalovirus. Curr Opin Virol 16: 63-69.

28. Ritzel RM, Crapser J, Patel AR, et al. (2016): Age-associated resident memory CD8 T cells in the central nervous system are primed to potentiate inflammation after ischemic brain injury. J Immunol 196: 3318-3330.

29. Mackay LK, Kallies A (2016): Transcriptional regulation of tissue-resident lymphocytes. Trends Immunol 38: 94-103.

30. Turner DL, Farber DL (2014): Mucosal resident memory CD4 T cells in protection and immunopathology. Front Immunol 5: 331.

31. Glennie ND, Yeramilli VA, Beiting DP, et al. (2015): Skin-resident memory CD4+ T cells enhance protection against Leishmania major infection. J Exp. Med 212: 14051414.

32. Teijaro JR, Turner D, Pham Q, et al. (2011): Cutting edge: tissue-retentive lung memor CD4 T cells mediate optimal protection to respiratory virus infection. J Immunol 187: 55105514.

33. Turner DL, Bickham KL, Thome JJ, et al. (2014): Lung niches for the generation and maintenance of tissue-resident memory T cells. Mucosal Immunol 7: 501-510.

34. Adachi T, Kobayashi T, Sugihara E, et al. (2015): Hair-follicle-derived IL-17 and IL-15 mediate skin-resident memory T cell homeostasis and lymphoma. Nat Med 21: 1272-1279.

35. Di Rosa F, Gebhardt T (2016): Bone marrow T cells and the integrated functions of recirculaying and tissue-resient memory T cells. Front Immunol 7: 1-13.

36. Park CO, Kupper TS (2015): The emerging role of resident memory $\mathrm{T}$ cells in protective immunity and inflammatory disease. Nat Med 21: 688-697.

37. Adamiak M, Tokarz-Deptuła B, Deptuła W (2014): Charakterystyka naturalnych komórek limfoidalnych (ILC). Postepy Hig Med Dosw 68: 1464-1471.

38. Martinez-Gonzalez I, Matha L, Steer A., Tahei F (2017): Immunological memory of group 2 innate lymphoid cells. Trends Immunol 38: 423-431.

39. Laidlaw BJ, Craft JE, Kaech SM (2016): The multifaced role of $\mathrm{CD}^{+} \mathrm{T}$ cells in $\mathrm{CD}^{+} \mathrm{T}$ cell memory. Nat Rev Immunol 16: 102-111.

40. Soerens AG, Costa AD, Lind JM (2016): Regulatory T cells are essential to promote proper CD4 T-cell priming upon mucosal infection. Mucosal Immunol 9: 1395-1406.

41. Vignali DAA, Collison LW, Workman CJ (2008): How regulatory T cells work. Nat Rev Immunol 8: 523-532. 
42. Chen X, Du Y, Lin X, et al. (2016): CD4+CD25+ regulatory T cells in tumor immunity. Int Immunopharmacol 34: 244-249.

43. Ito T, Yamada A, Batal I, et al. (2016): The limits of linked suppression for regulatory T cells. Front Immunol 7: 1-4.

44. Su J, Xie Q, Xu Y, et al. (2015): Role of CD8+ regulatory $\mathrm{T}$ cells in organ transplantation. Burns\&Trauma 2: 18-23.

45. Schmitt EG, Williams CB (2013): Generation and function of induced regulatory T cells. Front Immunol 4: 152.

46. Hilbrands R, Chen Y, Kendal AR, et al. (2016): Induced Foxp3+ T cells colonizing tolerated allografts exhibit the hypomethylation pattern typical of mature regulation T cells. Front Immunol 7: 1-8.

47. Tanoue T, Atarashi K, Honda K (2016): Development and maintenance of intestinal regulatory T cells. Nat Rev Immunol 16: 295-309.

48. Ng WF, Duggan PJ, Ponchel F, et al. (2001): Human CD4+CD25+ cells: a naturally occurrting populations of regulatory T cells. Blood 98: 2736-2744.

49. Vinay DS, Kim CH, Choi BK, Kwon BS (2009): Origins and functional basis of regulatory $\mathrm{CD} 11 \mathrm{c}^{+} \mathrm{CD}^{+} \mathrm{T}$ cells. Eur J Immunol 39: 1552-1563.

50. Sanchez AM, Yang Y (2011): The role of natural regulatory $\mathrm{T}$ cells in infection. Immunol Res 49: 124-134.

51. Feuerer M, Hill JA, Mathis D, Benoist C (2009): Foxp3+ regulatory T cells: differentiation, specificification, subphenotypes. Nat Immunol 10: 689-696.

52. Wing K, Sakaguchi S (2010): Regulatory T cells exert checks and balances on self-tolerance and autoimmunity. Nat Immunol 11: 7-13.

53. Sun L, Jin H, Li H (2016): GARP: a surface molecule of regulatory $\mathrm{T}$ cells that is involved in the regulatory function and TGF $\beta$ releasing. Oncotarget 7: 42826-42836.

54. Chen Z, Han Y, Gu Y, et al. (2013): Cd11 $\mathrm{c}^{\text {high }} \mathrm{CD} 8+$ regulatory $\mathrm{T}$ cell feedbach inhibits CD4 T cell Immune response via Fas ligand - Fas pathway. J Immunol 190: 6145-6154.

55. Churlaud G, Pitoiset F, Jebbawi F, et al. (2015): Human and mouse $\mathrm{CD} 8+\mathrm{CD} 25+\mathrm{FOXP} 3+$ regulatory $\mathrm{T}$ cells at steady state during interleukin-2 therapy. Front Immunol 6: 1-10.

56. Akane K, Kojima S, Mak TW, et al. (2016): CD8+C$\mathrm{D} 122+\mathrm{CD} 49 \mathrm{~d}^{\text {low }}$ regulatory $\mathrm{T}$ cells maintain T-cell homeostasis by killing activated $\mathrm{T}$ cells via Fas/FasL-mediated cytotoxicity. Proc Natl Acad Sci USA 113: 2460-2465.

57. Liu J, Chen D, Nie GD, Dai Z (2015): CD8+CD122+ T-cells: a newly emerging regulator with central memory cell phenotypes. Front Immunol 6: 1-6.

58. Suzuki H, Shi Z, Okuno Y, Isobe K (2008): Are CD8+CD122+ cells regulatory T cells or memory T cells? Hum Immunol 69: 751-754.

59. Dai H, Wan N, Zhang S, et al. (2010): Cutting edge: programmed death- 1 defines $\mathrm{CD} 8+\mathrm{CD} 122+\mathrm{T}$ cells as regulatory versus memory T cells. J Immunol 185: 803-807.

60. Li S, Xie Q, Zeng Y, et al. (2014): A naturally occurring CD8+CD122+ T-cell subset as a memory-like Treg family. Cell Mol Immunol 11: 326-331.

61. Gratz IK, Truong HA, Yang SH, et al. (2013): Cutting edge: memory regulatory $\mathrm{T}$ cells require IL-7 and not IL-2 for their maintenance in peripheral tissues. J Immunol 190: 4483-4487.

62. Sanchez Rodriguez R, Pauli ML, Neuhaus IM, et al. (2014): Memory regulatory $\mathrm{T}$ cells reside in human skin. J Clin Invest 124: 1027-1036.

63. Vukmanovic-Stejic M, Zhang Y, Cook JE, et al. (2006): Human $\mathrm{CD} 4+\mathrm{CD} 25^{\text {hi }} \mathrm{Foxp} 3+$ regulatory $\mathrm{T}$ cells are derived by rapid turnover of memory populations in vivo. J Clin Invest 116: 2423-2433.
64. Guo Z, Jang MH, Otani K, et al. (2008): CD4+CD25+ regulatory $\mathrm{T}$ cells in the small intestinal lamina propria show an effector/memory phenotype. Int Immunol 20: 307-315.

65. Sanchez AM, Zhu J, Huang X, Yang Y (2012): The development and function of memory regulatory $\mathrm{T}$ cells after acute viral infections. J Immunol 189: 2805-2814.

66. Gratz IK, Campbell DJ (2014): Organ-specific and memory Treg cells: specifity, development, function and maintance. Front Immunol 5: 51-67.

67. Rosenblum MD, Way SS, Abbas AK (2016): Regulatory T cell memory. Nat Rev Immunol 16: 90-101.

68. Rowe JH, Ertelt JM, Xin L, Way SS (2012): Pregnancy imprints regulatory memory that sustains anergy to fetal antigen. Nature 490: 102-106.

69. Kinder JM, Jiang TT, Clark DR, et al. (2014): Pregnancy induced maternal regulatory $\mathrm{T}$ cells, bona fide memory or maintenance by antigenic reminder from fetal cell microchimerism? Chimerism 5: 16-19.

70. Ruocco MG, Chaouat G, Florez L, et al. (2014): Regulatory T-cells in pregnancy: historical perspective, state of the art, and burning questions. Front Immunol 5: 1-10.

71. Tsiantoulas D, Diehl CJ, Witztum JL, Binder CJ (2014): B cells and humoral immunity in atherosclerosis. Circ Res 114: 17431756.

72. Zasada M, Rutkowska-Zapała M, Lenart M, Kwinta P (2016): Rola limfocytów IRA B w wybranych procesach zapalnych. Post Hig Med Dosw 70: 164-199.

73. Rosser EC, Mauri C (2015): Regulatory B cells: origin, phenotype, and function. Immunity 42: 607-612.

74. Mauri C, Menon M (2015): The expanding family of regulatory B cells. Int Immunol 27: 479-486.

75. Sattler S, van der Vlugt LEPM, Hussaarts L: Regulatory B cells - implications in autoimmune and allergic disorders. In: Recent Advances in Immunology to Target Cancer, Inflammation and Infections, Kanwar JR (eds.). InTech 2012: 177-200.

76. Guzman-Genuino RM, Diener KR (2017): Regulatory B cells in pregnancy: lessons from autoimmunity, graft tolerance, and cancer. Front Immunol 8: 172.

77. Dörner T, Radbruch A (2007): Antibodies and B cell memory in viral immunity. Immunity 27: 384-392.

78. Kurosaki T, Kometani K, Ise W (2015): Memory B cells. Nat Rev Immunology 15: 149-159.

79. Tangye SG, Tarlinton DM (2009): Memory B cells: Effectors of long-lived immune responses. Eur J Immunol 39: 2065-2075.

80. Qi H (2016): T follicular helper cells in space-time. Nat Rev Immunol 16: 612-625.

81. Baumgarth $\mathrm{N}$ (2016): B-1 cell heterogeneity and the regulation of natural and antigen-induced IgM production. Front Immunol 7: 1-9.

82. Born WK, Huang Y, Zeng W, et al. (2016): A special connection between $\gamma \delta \mathrm{T}$ cells and natural antibodies? Arch Imunol Ther Exp 64: 455-462.

83. Rothstein TL (2016): Natural antibodies as rheostats for susceptibility to chronic diseases in the aged. Front Immunol 7: 1-7.

84. Savage HP, Baumgarth N (2015): Characteristics of natural antibody - secreting cells. Ann N.Y. Acad Sci 1362: 132-142.

85. Zan H, Casali P (2015): Epigenetics of peripheral B-cell differentiation and the antibody response. Front Immunol 6: 631.

86. Bar-Or A, Oliveira EML, Anderson DE, et al. (2001): Immunological memory: Contribution of memory B cells expressing costimulatory molecules in the resting state. J Immunol 167: 5669-5677. 
87. Stone SL, Lund FE (2016): IgM memory cells: first responders in malaria. Immunity 45: 235-237.

88. Lindner C, Thomsen I, Wahl B, et al. (2015): Diversification of memory B cells drives the continuous adaptation of secretory antibodies to gut microbiota. Nat Immunol 16: 880-888.

89. Onodera T, Takahashi Y, Yokoi Y, et al. (2012): Memory B cells in the lung participate in protective humoral immune responses to pulmonary influenza virus reinfection. Proc Natl Acad Sci USA 109: 2485-2490.

90. Li H, Borrego F, Nagata S, Tolnaz M (2016): Fc receptor-like 5 expression distinguishes two distinct subsets of human circulating tissue-like memory B cells. J Immunol 196: 4064-4074.

91. Barrington RA, Pozdnyakova O, Zafari MR, et al. (2002): B lymphocyte memory: Role of stromal cell complement and FcyRIIB receptors. J Exp Med 196: 1189-1199.

92. Lazarevic V, Glimcher LH, Lord GM (2013): T-bet: a bridge between innate and adaptive immunity. Nat Rev Immunol 13: 777-789.

93. Geiger TL, Sun JC (2016): Development and maturation of natural killer cells. Curr Opin Immunol 39: 82-89.

94. Vivier E, Raulet DH, Moretta A, et al. (2011): Innate or adaptive immunity? The example of natural killer cells. Science 331: 44-49.

95. Vivier E, Tomasello E, Bartin M, et al. (2008): Functions of natural killer cells. Nat Immunol 9: 503-510.

96. Niedźwiedzka-Rystwej P, Herberg M, Deptuła W (2012): Biology and role of NK cells selected data. Centr Eur J Immunol 37: 399-404.

97. Moretta L, Montaldo E, Vacca P, et al. (2014): Human Natural Killer cells: origin, receptors, function, and clinical application. Int Arch. Allergy Immunol 164: 253-264.

98. Della Chiesa M, Pesce S, Muccio L, et al. (2016): Features of Memory-Like and PD- $1^{+}$human NK cells subsets. Front Immunol 7: 1-8.

99. Björkström NK, Ljunggren HG, Michaëlsson J (2016): Emerging insights into natural killer cells in human peripheral tissues. Nat Rev Immunol 16: 310-320.

100. Marcus A, Raulet DH (2013): Evidence for natural killer cell memory. Curr Biol 23: R817-820.

101. O'Sullivan TE, Sun JC, Lanier LL (2015): Natural killer cell memory. Immunity 43: 634-645.

102. Gardiner CM, Mills KHG (2016): The cells that mediate innate immune memory and their functional significance in inflammatory and infectious disease. Semin Immunol 28: 343-350.

103. O'Leary JG, Goodarzi M, Drayton DL, von Andrian UH (2006): T cell- and B cell-independent adaptive immunity mediated by natural killer cells. Nat Immunol 7: 507-516.

104. Watzl C, Urlaub D, Fasenbender F, Claus M (2014): Natural killer cell regulation - beyond the receptors. F1000Prime Rep 6: 87.

105. Cerwenka A, Lanier LL (2016): Natural killer cell memory in infection, inflammation and cancer. Nat Rev Immunol 16: 112-123.

106. Min-Oo G, Kamimura Y, Hendricks DW, et al. (2013): Natural killer cells: walking three paths down memory lane. Trends Immunol 34: 251-258.

107. Sun JC, Beike JN, Lanier LL (2009): Adaptive immune features of natural killer cells. Nature 457: 557-561.

108. Hamon MA, Quintin J (2016): Innate immune memory in mammals. Semin Immunol 28: 351-358.

109. Holmes TD, Bryceson YT (2016): Natural killer cell memory in context. Semin Immunol 28: 368-376.
110. Sun JC, Beike JN, Lanier LL (2010): Immune memory redefined: characterizing the longevity of natural killer cells. Immunol Rev 236: 83-94.

111. Sun JC, Lopez-Verges S, Kim CC, et al. (2011): NK cells and immune ,memory”. J Immunol 186: 1891-1897.

112. Paust S, Gill HS, Wang BZ, et al. (2010): Critical role for the chemokine receptor CXCR6 in NK cell-mediated antigen-specific memory of haptens and viruses. Nat. Immunol 11: 1127-1135.

113. Paust S, von Andrian UH (2011): Natural killer cell memory. Nat Immunol 12: 500-508.

114. Fu X, Liu Y, Li L, et al. (2011): Human natural killer cells expressing the memory - associated marker CD45RO from tuberculous pleurisy respond more strongly and rapidly than CD45RO- natural killer cells following stimulation with interleukin-12. Immunology 134: 42-49.

115. Cooper MA, Elliot JM, Keyel PA, et al. (2009): Cytokine-induced memory-like natural killer cells. Proc Natl Acad Sci USA 106: 1915-1919.

116. Rölle A, Pollmann J, Cerwenka A (2013): Memory of infections: an emerging role for natural killer cells. PLoS Pathog 9: e1003548. 